\title{
Robust Stability for Neural Network with Discrete and Distributed Delays
}

\author{
Hongxia Sun \\ College of Science, Yanshan University, Qinhuangdao 066004, China \\ E-mail: watermoonholesky@sina.com \\ Yiming Chen \\ College of Science, Yanshan University, Qinhuangdao 066004, China \\ Wei Guan \\ College of Science, Yanshan University, Qinhuangdao 066004, China \\ Long Yu \\ College of Science, Yanshan University, Qinhuangdao 066004, China
}

Supported by the Foundation for the natural science of He Bei province of China (2007000381)

\begin{abstract}
The robust stability for neutral network with discrete and distributed delays is devoted. The stability analysis problem is converted into a convex optimization problem, and a linear matrix inequality (LMI) approach is utilized to establish the sufficient condition for the globally robust stability. This condition can be easily checked by using the MATLAB LMI Toolbox.
\end{abstract}

Keywords: Time-delay system, Uncertainty, Stability, Linear matrix inequality, Neural networks

\section{Introduction}

Time-delays inevitably exist in neural network for various reasons, and it can induce chaos instability in the neural network. Therefore stability analysis for neural networks with time-delays has been an attractive subject of research in the past years a. It is worth noting that, although the signal propagation is sometimes instantaneous and can be modeled with discrete delays, it may also be distributed during a certain time period so that the distributed delays should be incorporated in the model. In other words it is often the case that the neural network model possesses both discrete and distributed delays.

In view of the importance of both discrete and distributed delays in modeling neural networks, the dynamics analysis problem for neural networks with discrete and distributed delays has received much attention. Chen Wenhua, Lu Xueming. (2006) reported that robust stability for neural network with discrete and distributed delays has been studied. However, the restriction of time-delay is less than 1. In this paper, based on Lyapunov stability and linear matrix inequality, delay-dependent robust stability is derived the result is obtained under mild conditions, assuming neither differentiability nor monotony for activation function. This condition can be easily checked by using the MATLAB LMI Toolbox.

\section{Problem Formulations}

Considering the following neural networks with discrete and distributed delays

$$
\begin{gathered}
x(t)=-(K+\Delta K(t)) x(t)+(A+\Delta A(t)) f(x(t)-d(t)))+ \\
(B+\Delta B(t)) \int_{t-d(t)}^{t} f(x(s)) d s
\end{gathered}
$$


Where $x(t)=\left(x_{1}(t), x_{2}(t), \cdots, x_{n}(t)\right)^{T} \in R^{n}$ is the neuron state vector; $K=\operatorname{diag}\left(k_{1}, k_{2}, \cdots, k_{n}\right)$ is a diagonal matrix with $k_{i}>0,(i=1,2, \cdots, n) ; A=\left(a_{i j}\right)_{n \times n} B=\left(b_{i j}\right)_{n \times n}$ are the constant matrices with appropriate dimensions; $d(t)$ denote the time-varying delay satisfies $0 \leq d(t) \leq d, d(t) \leq \mu$,in which $d, \mu$ are the constants; neuron activation functions is presented by $f(x(t))=\left(f_{1}\left(x_{1}(t)\right), f_{2}\left(x_{2}(t)\right), \cdots, f_{n}\left(x_{n}(t)\right)^{T}\right.$.

Assumption 1 In system (1) $\Delta K(t), \Delta A(t), \Delta B(t)$ satisfies

$$
\Delta K(t)=D_{1} F_{1}(t) E_{1} ; \quad \Delta A(t)=D_{2} F_{2}(t) E_{2} ; \quad \Delta B(t)=D_{3} F_{3}(t) E_{3}
$$

In which $F_{i}(t)(i=1,2,3)$ satisfies $F_{i}^{T}(t) F_{i}(t) \leq I$.

Assumption $2 \forall i \in\{1,2, \cdots, n\}, f_{i}(s)$ satisfies $l_{i}^{-} \leq \frac{f_{i}\left(s_{1}\right)-f_{i}\left(s_{2}\right)}{s_{1}-s_{2}} \leq l_{i}^{+}$, in which $l_{i}^{-}, l_{i}^{+}$are the constants.

Base on the Brouwer's, equilibrium point will be moved to the origin

$$
\dot{y}(t)=-(K+\Delta K(t)) y(t)+(A+\Delta A(t)) g(y(t-d(t)))+(B+\Delta B) \int_{t-d(t)}^{t} g(y(s)) d s
$$

\section{Main Results}

Theorem1 Define $L_{1}=\operatorname{diag}\left(l_{1}^{-} l_{1}^{+}, \cdots l_{2}^{-} l_{2}^{+}\right), L_{2}=\operatorname{diag}\left(l_{1}^{-}+l_{1}^{+}, \cdots, l_{n}^{-}+l_{n}^{+}\right)$, for any given $0 \leq d(t) \leq d$, if there exist matrices $P>0, R>0, Q_{1}>0, Q_{2}>0$,positive definite diagonal matrix $U_{1}=\operatorname{diag}\left(u_{11}, \cdots, u_{1 n}\right), U_{2}=\operatorname{diag}\left(u_{21}, \cdots, u_{2 n}\right)$ and some appropriately matrices $X_{i}(i=1,2,3), Y_{i}(i=1,2,3)$, makes the establishment of the following matrix inequality, then system (2) In the equilibrium is globally asymptotically stable

$\Xi=\left(\begin{array}{ccccccccc}\psi_{11} & \psi_{12} & -X_{1}+X_{3}^{T} & Y_{1} A+L_{2} U_{2} & Y_{1} B & -d X_{1} & Y_{1} E_{1} & Y_{1} E_{2} & Y_{1} E_{3} \\ * & \psi_{22} & -X_{2} & U_{1}+Y_{2} A & Y_{2} B & -d X_{2} & Y_{2} E_{1} & Y_{2} E_{2} & Y_{2} E_{3} \\ * & * & \psi_{33} & 0 & L_{2} U_{3} & -d X_{3} & 0 & 0 & 0 \\ * & * & * & \psi_{44} & 0 & 0 & 0 & 0 & 0 \\ * & * & * & * & \psi_{55} & 0 & 0 & 0 & 0 \\ * & * & * & * & * & -d R & 0 & 0 & 0 \\ * & * & * & * & * & * & -\varepsilon_{1} I & 0 & 0 \\ * & * & * & * & * & * & * & -\varepsilon_{2} I & 0 \\ * & * & * & * & * & * & * & * & -\varepsilon_{3} I\end{array}\right)<0$

Where

$$
\begin{aligned}
& \psi_{11}=Q_{1}+X_{1}+X_{1}^{T}-Y_{1} K-K^{T} Y_{1}^{T}-2 L_{1} U_{2}+\varepsilon_{1} E_{1}^{T} E_{1} \\
& \psi_{12}=P-Y_{1}-K^{T} Y_{2}^{T}+X_{2}^{T} \\
& \psi_{22}=d R-Y_{2}-Y_{2}^{T} \\
& \psi_{33}=-(1-\mu) Q_{1}-X_{3}-X_{3}^{T}-2 L_{1} U_{3} \\
& \psi_{44}=Q_{2}-2 U_{2}+\varepsilon_{2} E_{2}^{T} E_{2} \\
& \psi_{55}=-(1-\mu) Q_{2}-2 U_{3}+\varepsilon_{3} E_{3}^{T} E_{3}
\end{aligned}
$$

*stand for Symmetry elements of matrix transpose

Proof Construct the Lyapunov-Krasovksii functional: 
$V(t)=V_{1}(t)+V_{2}(t)+V_{3}(t)+V_{4}(t)$

Where

$V_{1}(t)=y^{T}(t) P y(t)$

$V_{2}(t)=\int_{-d}^{0} \int_{t+\theta}^{t} y^{T}(s) R y(s) d s d \theta$

$V_{3}(t)=\int_{t-d(t)}^{t} g^{T}(y(s)) Q_{1} g(y(s)) d s$

$V_{4}(t)=\int_{t-d(t)}^{t}\left(\int_{s}^{t} g^{T}(y(\theta)) d \theta\right) Q_{2}\left(\int_{s}^{t} g(y(\theta)) d \theta\right) d s$

Now the derivative of $V(t)$ along the trajectories of system (2) yields

$\dot{V}_{1}(t)=2 y^{T}(t) P \dot{y}(t)=2 y^{T}(t) P\left(-K y(t)+A g(y(t-d(t)))+B \int_{t-d(t)}^{t} g(y(s)) d s\right)$

$\left.\dot{V}_{2}(t)=d \dot{y}^{T}(t) R \dot{y}(t)\right)-\int_{t-d}^{t} \dot{y}^{T}(s) R \dot{y}(s) d s$

$\dot{V}_{3}(t) \leq g^{T}(y(t)) Q_{1} g(y(t))-(1-\mu) g^{T}(y(t-d(t))) Q_{1} g(y(t-d(t)))$

$\dot{V}_{4}(t) \leq-\left(\int_{t-d}^{t} g^{T}(y(\theta)) d \theta\right) Q_{2}\left(\int_{t-d}^{t} g(y(\theta)) d \theta\right)+d g^{T}(y(t)) Q_{2} g(y(t))$

Base on Leibniz-Newton formula and Schur lemma, we can get

$\dot{V}(t)-2 \sum_{i=1}^{n} u_{1 i}\left(g_{i}\left(y_{i}(t)\right)-l_{i}^{-} y_{i}(t)\right)\left(g_{i}\left(y_{i}(t)\right)-l_{i}^{+} y_{i}(t)\right)$

$-2 \sum_{i=1}^{n} u_{2 i}\left(g_{i}\left(y_{i}(t-d(t))\right)-l_{i}^{-} y_{i}(t-d(t))\right)\left(g_{i}\left(y_{i}(t-d(t))\right)-l_{i}^{+} y_{i}(t-d(t))\right)$

$=\frac{1}{d(t)} \int_{t-d(t)}^{t} \eta^{T}(t, s) \Xi(t) \eta(t, s) d s<0$

Where

$$
\Xi(t)=\left(\begin{array}{cccccc}
\varphi_{11} & \varphi_{12} & -X_{1}+X_{3}^{T} & Y_{1} A+L U_{1} & Y_{1} B & -d(t) X_{1} \\
* & \varphi_{22} & -X_{2} & Y_{2} A & Y_{2} B & -d(t) X_{2} \\
* & * & \varphi_{33} & 0 & L_{2} U_{2} & -d(t) X_{3} \\
* & * & * & \varphi_{44} & 0 & 0 \\
* & * & * & * & \varphi_{55} & 0 \\
* & * & * & * & * & -d(t) R
\end{array}\right)<0
$$

Then $V(t)<0$, system (2) In the equilibrium is globally asymptotically stable.

\section{Numerical Examples}

Examples 1 Consider the following neural networks with discrete and distributed delays

$$
\dot{y}(t)=-K y(t)+A g(y(t)-d(t))+B \int_{t-d(t)}^{t} g(y(t))
$$

Where

$$
K=\left(\begin{array}{cc}
-1 & -2 \\
0 & 1
\end{array}\right), A=\left(\begin{array}{ll}
0 & 0 \\
0 & 1
\end{array}\right), B=\left(\begin{array}{ll}
-1 & 0 \\
-1 & 0
\end{array}\right)
$$


Ensure time-delay system (6) stability and the maximum lag time $d$ of progressive Park Ju H. (2006) and Song Qiankun, Cao Jinde. (2007) reported that $d=0.5658, d=0.74$. By theorem 1 , the method shows $d=1.7192$

And

$P=\left(\begin{array}{cc}41.85 & -1.36 \\ -1.36 & 1.26\end{array}\right) R=\left(\begin{array}{cc}9.44 & 13.94 \\ 13.94 & 23.12\end{array}\right) Q_{1}=\left(\begin{array}{cc}-1 & 0 \\ 0 & 0\end{array}\right) Q_{2}=\left(\begin{array}{cc}0 & 0 \\ 0 & -1\end{array}\right)$

Compared with some existing results, the criteria obtained in our paper are less conservative.

\section{Conclusion}

In this paper, by using the method of Lyapunov and LMI. One sufficient condition for neural networks with discrete and distributed delays is derived. Finally compared with some existing results, the criteria obtained in our paper are less conservative.

\section{References}

Chen Wenhua, Lu Xueming. (2006). Delay-dependent exponential Stability of Neural Networks with variable delay: an LMI approach. IEEE Transactions on Circuits and Systems-II: Express Briefs, 2006,53(9):837-842.

Park Ju H. (2006). On global stability criterion for neural networks with discrete and distributed delays. Chaos, Solitons and Fractals, 2006, 30(4):897-902.

Song Qiankun, Cao Jinde. (2007). Global exponential stability of bidirectional associative memory neural networks with distributed delays. Journal of Computational and Applied Mathematics, 2007,202 (2):266-279.

Song Qiankun, Cao Jinde. (2006). Stability analysis of Cohen-Grossberg neural networks with both time-varying and continuously distributed delays. Journal of Computational and Applied Mathematics, 2006, 197(1): 188-203. 